\title{
ESTADO DE LA CUESTIÓN
}

\section{SOBRE CIENCIA Y LITERATURA}

\author{
María M. García Lorenzo (ed.)
}





\title{
INTRODUCCIÓN
}

\author{
INTRODUCTION
}

\author{
María M. GARCÍA LORENZO \\ Universidad Nacional de Educación a Distancia \\ mgarcia@flog.uned.es
}

Resumen: Las páginas que siguen constituyen una presentación del monográfico sobre ciencia y literatura de la revista Signa. Pretenden, por un lado, proporcionar un repaso a la separación de prácticas y saberes (fruto de la necesidad de perfilar identidades culturales) desde la aparición de la ciencia moderna y, por otro, mostrar distintos modos de hibridación y cruce de influencias entre lo científico-tecnológico y lo literario, desde los que podemos aproximarnos a textos de diversos géneros. Por último, se presentan y contextualizan los artículos que componen este volumen.

Abstract: These pages constitute a presentation of Signa's monograph on science and literature. On the one hand, this introduction aims to offer an outline of the division of knowledge, which was the result of the need to chart cultural identities since the apparition of modern science. On the other, it shows several modes of hybridism and cross-fertilization between the scientific-technological and the literary, from which we can approach texts of different genres. Lastly, the articles composing this monograph are presented and contextualized. 
Palabras clave: Ciencia y literatura. Discurso cultural. «Dos culturas». Hibridación.

Key words: Science and literature. Cultural discourse. «Two cultures». Hybridism.

\section{DE CIENCIAS Y DE LETRAS}

Si la ciencia no se limita a la búsqueda y cuantificación del dato observado, si involucra también a la compleja y ambivalente condición humana; si, además, es posible en ella hablar también de belleza, armonía o estilos [...] y si en diversos y obvios sentidos para la mayoría la literatura se acerca mejor a la vida, a la realidad cotidiana y universal, a los sentimientos y pasiones, ¿por qué no recurrir también a ella para que la ciencia penetre en ámbitos culturales y sociales de los que tradicionalmente ha sido excluida, para que deje de tener sentido hablar de dos culturas separadas por un golfo de mutua incomprensión? (Sánchez Ron, 2000: 2-3).

La sección monográfica que aquí se presenta está dedicada a la literatura y la ciencia, o más concretamente al interés que despierta la ciencia (y sus derivaciones tecnológicas) entre autores de géneros diversos. El título reúne en una misma unidad lingüística dos categorías que, a priori, nos parecen tan distantes como en su día pudo parecer estudiar el texto literario en combinación con la historia, la psicología, las leyes o el cine. «Literatura y ciencia» suena más arriesgado, más rompedor, porque traspasa una línea que se ha ido fortaleciendo a lo largo de los siglos desde que la era moderna perfiló la ciencia (como conocimiento racional, objetivo, universal, a partir de la observación y análisis de datos) separadamente de la filosofía, y la literatura (como conjunto de obras imaginativas ligadas a la expresividad, la percepción y la emoción) de cualquier otra forma de escritura.

Pero la experiencia vital rechaza estas demarcaciones: «El mundo, recordemos, no conoce fronteras, es interdisciplinar. Las fronteras las ponemos nosotros» (Sánchez Ron, 2012). Como seres humanos y seres sociales buscamos respuestas a cuestiones grandes y pequeñas, modelos que nos expliquen cómo se organiza lo que nos encontramos a diario, parámetros que anticipen lo que nos puede acontecer y cómo estar preparados para ello. Partiendo de esta inquietud común de explorarnos por los cuatro costados y crear representaciones que nos ayuden a (sobre)vivir, la literatura y la ciencia tienen mucho que compartir. Italo Calvino nos presta sus palabras para 
explicar este encuentro: él detecta una tradición en la literatura europea en que el texto literario es como «a map of the world and of the knowable, of writing driven by a thirst for knowledge that may by turns be theological, speculative, magical, encyclopedic, or may be concerned with magical philosophy or with transfiguring, visionary observation» (1982: 29). Empecemos por tanto por el momento en que los saberes sobre lo humano y lo no humano se especializan.

Lo que denominamos «conocimiento» varía histórica y culturalmente. Nuestro modo contemporáneo, racional, eurocéntrico, de construir conocimiento y aplicarlo a la sociedad proviene de momentos históricos y socioculturales muy concretos, la Reforma y la Ilustración, cuando el racionalismo surge como un modelo específico de conceptualizar la existencia, adecuado para su contexto. Miguel de Asúa subraya esta dimensión histórica de la separación entre la actividad científica y la actividad auto-referencial y estética de la literatura (2004: 13), y señala que la «ciencia» tal como la entendemos hoy no existe antes del siglo XVI, pues el saber premoderno se acerca más a una filosofía de lo natural que a una sistematización del conocimiento sobre la naturaleza. La ciencia moderna de lo físico-natural nace a partir del Renacimiento en Europa. De hecho, fue Francis Bacon quien popularizó el término «ciencia». La revolución científica iniciada con el empirismo, derivada del individualismo humanista del siglo XVI y antesala de la Ilustración, es hija de la Reforma: el mundo material se consideraba una máquina inanimada, sin participación divina, cuyos misterios no se encontraban encerrados en textos sagrados reveladores sino en sí misma. La «nueva ciencia» contribuyó al fortalecimiento del Protestantismo ${ }^{1}$, pues la Iglesia de Roma basaba sus doctrinas principalmente en textos de la antigüedad clásica como los de Aristóteles y Platón. El siglo XVII, por tanto, fue testigo de un trasvase de poder y autoridad: de la interpretación de la palabra divina a la epistemología del texto natural. Cuando se institucionaliza el conocimiento en el siglo XVIII, en la estela del empirismo utilitario de Francis Bacon, se abre la brecha entre la cultura científica y la humanística. Esta ciencia ya usa el nombre por el que la conocemos hoy y está a punto de establecer el título y la profesionalidad del «científico» (del inglés scientist, acuñado por William Whewell en 1844 (Asúa, 2004: 13-14)).

1 Merrison (en Shaffer, 1998: 171-172) recuerda que el empirismo fue no sólo una revolución científica sino un discurso identitario inglés que minimizaba el pensamiento extranjero (la filosofía clásica mediterránea, el cartesianismo francés...) o no anglicano (pagano, católico...). 
Pero esa ciencia de Bacon y Descartes, que también abarcaría la química, la física, la medicina, la botánica, la astronomía y los sublenguajes matemáticos, es también contemporánea de la literatura moderna de Cervantes (Kundera, 1987: 5) y, como apunta Charlotte Sleigh, la novela y el microscopio son dos tecnologías que aparecieron casi al tiempo (2011: 11), aunando simbólicamente la inspección de lo material. El sujeto literario y el sujeto científico van de la mano porque se construyen a la vez. La exploración del ser humano a través del texto literario en sus diversas manifestaciones contribuyó considerablemente a la construcción del ser humano como objeto de conocimiento. Nociones como las de qué somos, cómo esperamos ser, cómo nos definimos en situaciones determinadas, qué relación guardamos con el entorno, cómo nos percibimos o cómo vemos a los demás pusieron sobre la mesa los temas, actitudes y objetivos que progresivamente dieron lugar a los métodos y las prácticas de diversas disciplinas científicas. Las fuerzas constructivas de la literatura y la ciencia ponen al ser humano en relación con lo natural, lo cultural, lo divino, lo mismo o el Otro, y contribuyen a que lo leamos de determinada forma.

Aunque los docentes universitarios estamos acostumbrados a las cinco ramas de conocimiento que agrupan los saberes (Artes y Humanidades, Ciencias, Ciencias Sociales y Jurídicas, Ciencias de la Salud y Arquitectura e Ingeniería), nuestra sociedad aún percibe «dos culturas» diferenciadas, las ciencias y las letras, que parecen discurrir apaciblemente paralelas. Aunque nadie pone en duda que la vida es interdisciplinar, los desencuentros (a veces enfrentamientos abiertos) entre saberes se han sucedido a lo largo de los últimos siglos. Por ejemplo, a finales del siglo XIX surge la polémica entre la educación científica y la educación clásica entre Thomas Huxley ( «Science and Culture», conferencia pronunciada en 1880 anunciando la inevitabilidad de la supremacía científica y abogando por una educación más inclusiva) y Matthew Arnold (quien le dio réplica en su charla «Literature and Science» de 1882, donde ampliaba el término «literatura» para incluir tratados de ciencias naturales clásicos como Principia de Newton o El origen de las especies de Darwin, y donde defendía que el conocimiento utilitario que proponía Huxley no lleva a juicios sobre conducta o belleza). Según aprecia O'Connor (2007: 14-15), esta controversia se basa en la percepción de que tal separación es mayor de lo que en realidad fue en su momento, motivada sobre todo por la necesidad de demarcar identidades culturales y profesionales. La educación tradicional, sobre todo en el ámbito anglosajón, ciertamente favorecía las pedagogías basadas en pensadores, escritores e historiadores clásicos, más que en prácticas científicas. Como indica Gillian Beer 
(2010), este tipo de educación robustecía y conformaba «the way people set about thinking (and thinking about thinking)». No era extraño encontrar alusiones o citas a autores clásicos en los ensayos de los científicos, una costumbre que situaba culturalmente al autor, encuadrándolo en un contexto educativo adecuado, compartido por lectores igualmente instruidos, que lo legitimaba (Geer, 2010). La autoridad científica, por tanto, llegaba de la mano de una tradición humanista que le aportaba soporte lingüístico, refrendo cultural, y certificación de clase.

El debate entre Arnold y Huxley fue duplicado ya en el siglo XX entre C. P. Snow (su conferencia dedicada a las «dos culturas» tuvo lugar en 1959) y F. R. Leavis (quien le respondió con «The Two Cultures? The Significance of C. P. Snow» en 1962). El físico y novelista Snow lamentaba la separación de las dos actividades y la ignorancia mutua, que dificultaba la aplicación global de la ciencia como esperanza del futuro de la humanidad. El crítico literario Leavis respondió, entre otras cosas, que la ciencia no constituye una cultura ni aporta moral, dirección o significado, aspectos que pueden en cambio ser abordados por la literatura. Lo que en un principio encerraba una distinción puntual y local entre sistemas de aprendizaje se dilató hasta infiltrarse en nuestro modo de definirnos («de ciencias» o «de letras») y, cosa más seria, llegó a plantear debates más agrios entre defensores y detractores de la actividad científica, como se explica a continuación.

La «guerra de las ciencias» tuvo lugar en los años 90 del siglo XX cuando un colectivo de científicos se indignó ante la desacreditación de la ciencia en el pensamiento postestructuralista, feminista y multicultural (Gross y Levitt, 1995: 4-5). A sus ojos, la objetividad de la ciencia, su método, y su capacidad para observar, analizar y representar la realidad quedaba en entredicho cuando los investigadores en estudios culturales y sociales empleaban términos como «construcción social» o acercamientos relativistas a los motivos, objetivos o procedimientos científicos, en lo que parecía ser un posicionamiento anticientífico. Gross y Levitt lamentaban la hostilidad hacia los orígenes intelectuales de la ciencia moderna a manos de académicos que desconocen sus principios básicos, y denunciaban el peligro de la interdisciplinariedad cuando se manejan conceptos científicos sin la formación necesaria. Inspirado por Gross y Levitt, el físico Alan Sokal intentó demostrar la falacia subyacente a este trasvase mal entendido con la publicación de dos artículos (1996a y 1996b): el primero de ellos, aparecido en Social Text, hacía una defensa de la gravedad cuántica como constructo lingüístico, mientras el segundo, publicado en Lingua Franca, exponía la parodia del anterior (un texto «salted with nonsense», en sus propias palabras, pero en 
línea con teorías de pensadores postmodernos como Jean-François Lyotard o Jaques Derrida) ${ }^{2}$.

Ninguna realidad existe fuera del lenguaje que la produce, y tanto ciencia como literatura son expresiones de una cultura de la que forman parte. El acercamiento posestructuralista cuestiona la autoridad y el poder del discurso científico al enmarcarlo en estructuras sociales y culturales que funcionan en todos los ámbitos de la vida. Descubrir y analizar estas estructuras en medicina, sociología, antropología, o en el texto literario no subvierte necesariamente los logros de la ciencia, cuyos resultados quedan validados por sus métodos.

Algunos intentan superar esta separación proponiendo una cultura única (o una «tercera cultura», como sugiere Elinor Shaffer, 1998) que aúne ambos discursos y defienda la relevancia de ambos. El mismo Snow, en la reedición de «The Two Cultures» de 1963, anotaba que tal cultura ya se estaba gestando más allá de la educación clásica, y que «we can repair communications to some extent» si conseguimos educar:

a large proportion of our better minds so that they are not ignorant of imaginative experience, both in the arts and in science, nor ignorant neither of the endowments of applied science, of the remediable suffering of most of their fellow humans, and of the responsibilities which, once they are seen, cannot be denied (Snow, 1998: 100)․

Hoy en día, los estudios interdisciplinares están consolidados en la academia y acogen diversos modos de encuentro entre ciencias y letras, un encuentro basado en la naturaleza discursiva de toda forma de cultura. La estructura de las revoluciones científicas, de Thomas Khun (1962), postulaba que la historia de la ciencia se basa más en paradigmas sucesivos (culturalmente determinados y con influencia en las premisas y objetivos de los científicos) que en la linealidad de la actividad científica. Khun es un punto de referencia en el acercamiento de las ciencias naturales y las sociales al poner el acento en el relativismo sociológico e histórico de los avances científicos.

2 Puede consultarse el artículo de Blanco (2001) para una narración detallada y contextualizada de la polémica.

3 En la estela de algún modelo británico, García Aranda y González Agudelo (2007) proponen utilizar la literatura como medio para enseñar contenido relacionado con las ciencias naturales y para desarrollar el pensamiento científico. 
Estos debates nos han ayudado a mirar la obra literaria en profundidad, multiplicando sus lecturas: la teoría de cuerdas en las narraciones de Jorge Luis Borges, el behaviorismo en Ernest Hemingway, la geología en Juan Benet, la cosmología en la poesía barroca, la novela realista y la evolución de las especies, la ecología y la literatura naturalista.

Para los escépticos que detectan más distancia que acercamiento entre campos, el argumento del lenguaje o el estilo es muy poderoso. No es sencillo encontrar consenso en cuanto a los rasgos distintivos de un estilo decididamente literario, ni en cuanto a que la falta consciente de estilo en busca de neutralidad científica puede constituir un estilo en sí mismo susceptible de análisis literario (Sleigh, 2011: 9) ${ }^{4}$. Ningún hecho científico escapa a su representación subjetiva, generalmente lingüística -Carol Reeves afirma que sin lenguaje no existirían los hechos, puesto que no podemos escapar a las palabras (2005: 10)-. Lo que esperanzadamente denominamos «lenguaje científico» deriva sus significados de un entramado de signos compartidos por todos, y solo es significativo en cuanto a su relación con esta superestructura lingüística y cultural. Cuando Barthes propone que la ciencia no puede reclamarse como un «código superior» (Barthes, 2009: 20) en base a la transparencia de su lenguaje, Italo Calvino duda de esta visión simplista y de que la ciencia sea tan «compacta» y «segura de sí misma» (1982: 29) como declara Barthes. En este sentido es especialmente agudo el trabajo de Gillian Beer (1985), quien estudió El origen de las especies como literatura, explorando la interacción entre lenguaje y conocimiento en el trabajo darwiniano. Beer examinó metáforas como «evolución», «árbol» y «herencia» (entre otras muchas) concluyendo que fue precisamente este lenguaje metafórico el que propició la repercusión futura de la obra, tanto en su contexto científico como en otros aparentemente más alejados, como el de la literatura: «His language does not close itself off authoritatively nor describe its own circumference. [...] The nature of his argument led into expansion, transformation, and redundancy of information. The Darwinian world is always capable of further description» (1985: 55, en cursiva en el original). Otros, como Pessoa, simplemente encuentran belleza en el texto científico: «El binomio de Newton es tan bello como la Venus de Milo» («El viento, afuera»)5, y para Calvino,

\footnotetext{
4 De hecho, existe todo un campo de estudio dedicado a la retórica de la ciencia en el que lingüistas, sociólogos y filósofos investigan el lenguaje de los textos científicos (difusión, debate, consenso, presentación de modelos, etc.) como persuasión.

5 Tampoco los científicos se desentienden del placer estético del mundo material o de su estudio: Fearful Symmetry:The Search for Beauty in Modern Physics (1987), de Anthony Zee (en español, Temerosa simetría: la búsqueda de la belleza en la física moderna, en Ellago), The Elegant Universe (2000)
} 
Galileo escribía su ciencia con un lenguaje conscientemente literario, expresivo, imaginativo e incluso lírico (1982: 30).

Decir que la ciencia influye en la literatura implica situar a la ciencia como referente de verdad y autoridad, como centro de la cultura más que como participante en la construcción de la misma. Como señala Katherine Hayles (1989: 317), este posicionamiento «ignores the ways in which scientific theories, no less than literary theories and literature, are social constructions that reflect the prevailing concerns of the culture». Podemos hablar, preferiblemente, de epistemes foucaultianos dominantes que prevalecen en determinados momentos históricos y modelan disciplinas dispares. Sostiene Shaffer que es esencial poner el foco en cómo la ciencia interactúa con otras disciplinas, puesto que es el discurso actual con más poder a nivel económico, social, político e incluso ideológico, pues ha reemplazado a la religión y a la filosofía en su labor de dar respuesta a antiguas preguntas trascendentales (1998: 2). Veremos también en la siguiente sección cómo el texto literario puede participar en la investigación científica y su aplicación tecnológica.

\section{EL ANÁLISIS LITERARIO SE ACERCA A LA CIENCIA ${ }^{6}$}

Como se comenta al principio de esta introducción, siempre se ha estudiado la literatura con relación a algo más: la religión, el arte, la historia, las leyes, la psicología. Algunos terrenos nos parecen más afines, otros más distantes. Pero a veces la tecno-ciencia nos parece el campo opuesto, justo al otro lado de la línea (o del campus universitario), y ciertamente no tan cercano como los vecinos de la rama de Humanidades. La hibridación disciplinar comenzó a tomar forma a partir de los años 70, cuando el mapa global presentó cuestiones económicas, medioambientales, sociales y políticas que requerían un acercamiento plural, reñido con la fragmentación académica (Hayles, 1994: 28). Sánchez Ron califica la encrucijada de saberes de este nuevo siglo como «la nueva Ilustración», una red que ya caracterizó a los ilustrados del siglo XVIII, necesaria para la formación y el avance de nuestra sociedad. Ya advertía Ortega y Gasset sobre el riesgo de incultura

\footnotetext{
de Brian Greene (El universo elegante, en Crítica) y Symmetry and the Beautiful Universe (2004), de Leon Lederman y Christopher Hill (La simetría y la belleza del Universo, en Tusquets), transmiten nociones y modelos físicos desde la elegancia y la estética de la simetría en física, mientras sus títulos sugieren una idea de belleza cercana al canon renacentista y neoclásico.

6 El artículo de García Landa incluido (1991: 248) nos ofrece un detallado repaso a los «common grounds and interpenetrations» entre ciencia y literatura desde la teoría literaria.
} 
que puede devenir de la especialización científica. Robert Heinlein resulta algo más detallado en esta famosísima cita:

A human being should be able to change a diaper, plan an invasion, butcher a hog, conn a ship, design a building, write a sonnet, balance accounts, build a well, set a bone, comfort the dying, take orders, give orders, cooperate, act alone, solve equations, analyze a new problem, pitch manure, program a computer, cook a tasty meal, fight efficiently, die gallantly. Specialization is for insects (Heinlein, 1973: 248).

Cuando trasladamos esos trasvases al mundo académico nos encontramos con algunos escollos. El primero y más evidente es la compartimentación de estudios e investigaciones por razones de operatividad, pero además existen las tradiciones metodológicas de cada disciplina, es decir, cómo se produce y se estructura el conocimiento (Hayles, 1994: 27) a partir de diferentes interrogantes iniciales. La resignificación de términos entre ambos ámbitos es una de las ocupaciones de los estudios culturales actuales. Nuestro uso de teorías y términos científico-tecnológicos (ya sean células, entropías o saltos cuánticos) tiende a la estética, a la especulación, a entender cómo nos vemos y cómo nos interpretamos, cómo nos gustaría ser y cómo tememos ser. La ciencia nos abre puertas muy interesantes, y resulta difícil no intentar explorar las apasionantes posibilidades de lectura que nos brinda. Por ello, apunta Katherine Hayles, nuestro trabajo interdisciplinar no es verdaderamente tal, pues «the only way we can process another discipline is as a sub-category of our own» (1994: 30). Nuestro análisis filológico-cultural es una lectura de la ciencia en textos de diversa tipología, y en ningún caso entorpece o desprestigia el análisis científico. Debemos considerar también que la representación de la ciencia y la tecnología deriva de un conocimiento a nivel divulgativo por parte de los escritores, pues salvo excepciones (por dar dos ejemplos, Thomas Pynchon, que estudió ingeniería, o José Echeagaray, que era matemático) no leen revistas especializadas ni manejan las teorías científicas con soltura. Sin embargo, es ese mismo conocimiento el que llega a la sociedad, a sus grupos de presión, a la clase política y a sus votantes, a las instituciones que financian investigaciones, etc. Por ello resulta de interés comprobar qué imágenes de la ciencia y la tecnología se esconden en las páginas que leen, han leído o leerán millones de lectores.

Son varias las zonas de encuentro entre ciencia y literatura: la literatura como espejo de nociones científicas o avances tecnológicos, la literatura como vigía de las repercusiones éticas de la ciencia, la literatura como cor- 
pus de datos para el estudio científico, la literatura como anticipación científica, los modelos y conceptos científicos como recursos creativos o principios organizativos del texto. Un campo de estudio realmente fascinante es el de la anticipación científica, representado en estas páginas por el artículo de Margarita Rigal y Ricardo Marín, y la influencia del texto literario sobre el trabajo científico, que apuntan a que la «influencia» entre ciencia y literatura no es un trasvase unidireccional sino un camino de ida y vuelta entre lo científico-tecnológico por el método, el lenguaje, o las ideas. En La Ilíada de Homero, por ejemplo, ya encontramos una noción de materia inerte animada en los siervos de oro que construye Hefesto, antecedentes de los robots que poco a poco comienzan a ser una realidad entre nosotros. Igualmente sucede con los viajes aéreos (Rigal y Marín dan más detalles) y también con los viajes a la Luna, que quedaron inscritos en el desiderátum popular con ayuda de Cyrano de Bergerac y Jules Verne. Los laberintos y bifurcaciones borgianos se adelantan por su parte a las teorías sobre universos paralelos que se multiplican.

También existen casos más recientes que demuestran el trasvase de ideas entre la escritura creativa y la actividad científica. Uno de ellos lo encontramos en la novela Contact, del astrónomo Carl Sagan (1985). Sagan envió el manuscrito al físico Kip Thorne para que este comprobara los datos científicos del libro y su coherencia con las leyes físicas. Thorne le sugirió que descartara el viaje por el tejido espaciotemporal de la protagonista a través de un agujero negro, y que incluyera en cambio un agujero de gusano. La existencia de estos túneles tetradimensionales había sido sugerida por Albert Einstein a principios del siglo XX, pero fue a partir de este contacto (nunca mejor dicho) interdisciplinar entre literatura y ciencia, y entre astronomía y física, que una comunidad de físicos teóricos retomó la idea e inició el estudio de las deformaciones del espacio-tiempo. Si usted está leyendo una versión en línea de este monográfico, sepa que William Gibson creó el término ciberespacio para referirse a una forma de comunicación informatizada, una «alucinación consensuada», en Neuromancer (1984). En la reedición del año 2000, el epílogo de Jack Womack propone que tal vez la idea del ciberespacio que Gibson imaginó para la novela pudiera inspirar el modo en que finalmente se desarrolló Internet: «What if the act of writing it down, in fact, brought it about?» (Womack, 2000: 269) ${ }^{7}$.

7 El artículo electrónico «La ciencia de la literatura» contiene unos cuantos ejemplos interesantes sobre obras o escritores que tuvieron su relevancia en ciertos avances o descubrimientos científicos (Rodríguez Medina, 2013). 
Podemos preguntar directamente a los científicos y tecnólogos por los libros que los han influido de alguna manera. El editor de la revista MIT Technology Review, Jason Pontin, escribió un editorial, titulado «On Science Fiction: How it Influences the Imaginations of Technologists», en que comentaba cómo muchos tecnólogos llegan a su profesión desde la ciencia ficción, que también inspira la dirección que toma su trabajo. La revista Spectrum del IEEE (Institute of Electrical and Electronics Engineers) publicó en 2007 un pequeño reportaje sobre la literatura que más había inspirado a un grupo de ingenieros consultados. Aunque se podía esperar que la mayoría se decantara por la ciencia ficción, espacio «natural» de encuentro entre ciencias y letras, no es así (Zorpette y Ross, 2007) ${ }^{8}$.

Buena parte de los estudios literarios sobre la ciencia y la tecnología muestran cómo los autores desvelan las consecuencias nefastas de las aplicaciones científico-tecnológicas, presentando una imagen catastrofista de la ciencia como discurso cultural, como actividad profesional, o como origen de máquinas o procedimientos potencialmente (o comprobadamente) dañinos. Es frecuente que nos encontremos con castigos implacables al deseo de conocimiento (el mito creacional del Génesis y el mito clásico de Prometeo así nos lo cuentan, como también la historia del doctor Fausto). Las dudas sobre la solvencia moral de algunos científicos están presentes, por ejemplo, en la novela que inspiró la película Blade Runner, ¿Sueñan los androides con ovejas eléctricas? (donde la tecnología está al servicio de las corporaciones y el mercado) del gran agorero Philip K. Dick, o en la obra de teatro Los Físicos, de Friedich Dürrenmatt (donde tres internos de un manicomio, llamados Newton, Einstein y Möbius, debaten el destino del mundo), o en Oryx y Crake, de Margaret Atwood (donde un genetista ambicioso intenta reemplazar al ser humano actual por una versión más ecológica y pacífica). Sencillamente, el mal uso del descubrimiento científico o su aplicación, o la sensación de que puedan escaparse a un control cuya cara tampoco se muestra abiertamente, han producido algunas de las obras más descorazonadoras de los dos últimos siglos (París en el siglo XX de Julio Verne, Un mundo feliz de Aldous Huxley, 1984 de George Orwell o Los juegos del hambre de Suzanne Collins son cuatro distopías muy elocuentes).

8 Ya que el concepto de inspiración se menciona en los dos párrafos anteriores, vale la pena acudir al artículo de Valdés y Guyon en el volumen de Shaffer, The Third Culture: Literature and Science, donde explican el papel de la «educated imagination» (28) tanto en crítica literaria como en física. El artículo es resultado de las conversaciones mantenidas entre ambos investigadores a lo largo de años sobre cómo obtener sentido del texto poético y de ese otro texto que es el mundo físico. 
Muchas de las obras y películas contemporáneas, especialmente occidentales, ficcionalizan la ambigüedad hacia la máquina, una ambigüedad que no surgió tras la segunda Guerra Mundial pero que sí encontró en la posguerra un ambiente propicio. Por un lado, la amenaza nuclear fomentó el recelo hacia lo científico-tecnológico; por otro, los consumistas años 50 y 60 ensalzaron el hogar y la familia y, por tanto, hicieron un hueco a maravillas tecnológicas como la televisión o las lavadoras automáticas. La tecnofilia se incorporó pues al consumismo desatado, de modo que la amenaza de la tecnología quedó contrarrestada con la idea de seguridad asociada al hogar, de igual modo que en la actualidad la revolución tecnológica vinculada a la información se está asociando a ideas de libertad y democracia. Freese y Harris advierten un nuevo enfoque en el estudio de la literatura contemporánea, por el que un número de trabajos «attempt to relate literature to new information technologies in non-adversial ways» (2004: xiv). Reconociendo que vida, textos y tecnología se constituyen mutuamente en el complejo contexto en que nos encontramos, tal vez existan versiones de lo posthumano que amparen «the possibilities of information technologies without being seduced by fantasies of unlimited power and disembodied immortality» (Katherine Hayles, citada en Freese y Harris, 2004: xv).

Otra de las direcciones en que podemos leer la ciencia en el texto literario investiga cómo cada obra responde al episteme o marco del saber en su momento histórico y social. En este mismo volumen, en su artículo sobre el impacto de la ciencia en el siglo XIX europeo, Carlos Miguel Pueyo repasa las reacciones de los creadores románticos ante la supremacía de la ciencia. El Romanticismo es una época donde ya se había abandonado el paradigma antropomórfico de conocimiento en favor del empirismo, esto es, de dejar que el propio universo fuera el que hablara por sí mismo para poder conocerlo a través de sus manifestaciones visibles. Ante la abstracción analítica y cuantificadora de la ciencia, poetas y pensadores de la creación literaria como Shelley, Novalis y Goethe apostaban por la experiencia cualitativa. Las voces románticas expresaron sus recelos, intentando delimitar las actividades del sujeto poético y el sujeto científico, ya que se ocupaban del mismo objeto: el conocimiento del ser humano y su entorno. La literatura realista y naturalista del siglo XIX, por su parte, responde y contribuye a un modelo de conceptualización del mundo natural basado sobre todo en el juego de fuerzas y movimientos descrito por Isaac Newton, la termodinámica, el electromagnetismo, la evolución de las especies y los patrones de herencia de Mendel, entre otros estudios. La causalidad y la linealidad definían de igual modo el modelo newtoniano y la estética decimonónica. Los auto- 
res realistas contemplaron y describieron una sociedad donde lo científicotecnológico prometía progreso, como parecía anunciar la revolución industrial. Pero el discurso mecanicista y evolucionista que soportaba la consolidación capitalista en Occidente alimentaba diversos «ismos» (imperialismo, clasismo, racismo, patriarcalismo) en su diálogo con el darwinismo social. La literatura naturalista de Émile Zola, Theodore Dreiser o Thomas Hardy, entre otros, puso el foco en la representación y análisis de la experiencia humana en términos científicos. Zola, de hecho, proponía en Le roman experimental (1889) que la propia creación literaria se asemeja al método experimental, de modo que el escritor se convierte en un científico social. La observación de datos de estos «científicos» daba como resultado una narrativa en la que se viaja rápido, la electricidad transforma la relación que se establece con el entorno, se vive con cierta comodidad y seguridad, y las estructuras sociales se consolidan; a cambio, el ser humano como sujeto con voluntad y autonomía queda anulado.

El cambio epistémico del modelo dinámico newtoniano al modelo relativista se acompaña de la efervescencia artística modernista. El Modernismo fue una revolución intelectual y estética que coincidió en el tiempo con esta revisión que las ciencias hicieron de algunos conceptos fundamentales como la materia, el espacio, y el tiempo. La propia forma que empiezan a adoptar los textos literarios interpreta los nuevos principios físicos: la Teoría de la Relatividad aparece en 1916, y algunos de los hitos literarios experimentales del momento se publican poco después (The Waste Land de T. S. Eliot y Ulysses de James Joyce en 1922; Mrs Dalloway de Virginia Woolf en 1925). Por ejemplo, los escritores responden a la estimulante idea de la cuarta dimensión prestando gran atención a la representación del tiempo: abandonan la imagen del tiempo como algo lineal y absoluto, o como un marco de referencia inmutable donde situar los acontecimientos, y lo reemplazan por instantes que «duran» varias páginas, o acontecimientos narrados varias veces, o historias que dan saltos en el tiempo sin ajustarse a un orden cronológico. Tanto la física como la psicología y la filosofía del momento insisten también en que sujeto y objeto no existen de forma separada, y cualquier percepción de la naturaleza material es una construcción relativa de la mente. La fragmentación en forma y significado, sobre todo en la poesía de las primeras décadas del siglo XX, es una representación de la inevitable parcelación que ocurre en el acto de contemplar, conocer y entender, como sugiere el título del conocido poema de Wallace Stevens «Thirteen Ways of Looking at a Blackbird» (1917). El lenguaje modernista, tan consciente de sí mismo, refleja la necesidad de cuestionar el medio lingüístico como vehícu- 
lo de creación y transmisión de ideas, ya sean científicas, sociales, filosóficas, $\mathrm{o}$ artísticas.

Mientras la literatura del Modernismo correspondía a un paradigma de orden centralizado, el paradigma postmoderno acoge nociones de descentralización e inestabilidad que fomentan el rechazo de las metanarrativas. La nueva física del siglo XX trajo consigo una nueva metafísica, como lo demuestra que a veces encontremos denominaciones como «búsqueda del Santo Grial» para el estudio del genoma humano o «la partícula de Dios» para el bosón de Higgs. En el continuo espacio-tiempo, donde todo es energía (incluso el yo), el sujeto pierde sus límites. ¿Dónde queda pues nuestro sistema ideológico occidental, ese que traduce las verdades externas fidedignamente, que adjudica posiciones a todo y todos en el universo, porque todos sus componentes tienen su propia esencia y propósito, su lugar y su momento para existir? No nos puede extrañar que si la literatura del siglo XIX se impregnó de ideas sobre herencia y evolución, la del XX y XXI destile nociones sobre incertidumbre, incluso cuando numerosos científicos corregirían inmediatamente la acepción con que aquéllas se utilizan. El pensamiento postmoderno nos ha acostumbrado a derribar el sistema binario de opuestos cuya relación jerárquica predetermina el modo en que nos aproximamos a la experiencia. Una de estas oposiciones, la del caos y el orden, ha dado lugar a mucho encuentro en este territorio fronterizo entre ciencia y literatura. La ciencia interdisciplinaria del caos, con investigación en el campo de materias tan diversas como la meteorología, la geometría y la epidemiología, ha encontrado también su lugar entre las letras, tanto desde la crítica literaria como desde la propia creación literaria ${ }^{9}$. El contexto cultural en el que se contestan modelos legitimados y discursos de autoridad es un excelente caldo de cultivo para la revisión de los modelos newtonianos y euclidianos, y para la propuesta de nuevos paradigmas que permiten percibir estructuras universales en los sistemas caóticos. El cambio cultural que detecta Hayles con la aparición de la teoría del caos, la deconstrucción de Derrida o las arqueologías foucaultianas (Hayles, 1989: 313-314) responde al hecho de que todas las formas de pensamiento son construcciones culturales. La indeterminación del significado de un texto literario, la permeabilidad de este a infinitos

9 El artículo de Van Peer «Sense and Nonsense of Chaos Theory in Literary Studies» (en Shaffer, 1998 ) resulta interesante por su crítica a la investigación de la literatura con ayuda de la teoría del caos si aplicamos esta con todo rigor, pues estrictamente hablando ello implicaría aceptar que la literatura es un sistema caótico y, además, cerrado. 
textos pasados y futuros, y la incertidumbre causal sacuden antiguas nociones sobre el texto como sistema cerrado y predecible.

Tal vez echemos en falta que la ciencia nos proporcione, además de leyes y explicaciones, significados y parámetros morales (mar de fondo de los debates iniciales entre Arnold y Huxley), pero no podemos negar que la ciencia posee valores culturales:

The claim, for example, that empiricism can be unmarked, that is, can provide an evidentiary basis that «speaks for itself,» is after all a point of view, and one that may be held by science studies scholars as well as by scientists themselves. Moreover, it is a point of view with a history that establishes a cultural tradition: the tradition of «value-neutrality» or transparency. To distinguish between pure and applied knowledge, between hard and soft sciences invokes not only this value system, but the hierarchical nature of it, thus exemplifying the kind of cultural fact at issue here (Franklin, 1995: 166).

Del mismo modo que los conceptos, métodos y aplicaciones científicos proceden del contexto que lo generan, la ciencia también es agente en las relaciones de poder que se establecen cuando nombra, clasifica, equipara, excluye o incluye. Podemos aproximarnos a la ciencia en la literatura explorando el modo en que la ciencia predominante actúa en los márgenes del discurso, o cómo la ciencia afecta a los habitantes de esos márgenes. No conviene olvidar que el discurso médico y biológico ha sido utilizado a menudo para justificar la inferioridad del Otro, certificando sus supuestas limitaciones e incapacidades. Ya se ha mencionado la tradición educativa y cultural que conforma el pensamiento científico moderno, una tradición fundamentalmente blanca, masculina y eurocéntrica. Otras prácticas y creencias, también sistematizadas y orientadas al conocimiento del mundo material, suelen ser obviadas o relegadas a un segundo plano al no encajar en el planteamiento de «ciencia moderna» comúnmente aceptado. Por ello algunos historiadores y sociólogos de la ciencia, situados en posicionamientos revisionistas, plantean la identificación de «eurocentric and androcentric elements in the conceptual frameworks used to think about scientific and technological change, and in sciences and technologies themselves» (Harding, 1998: 18), a fin de expandir nuestro conocimiento del mundo natural y social, y de formular planteamientos, hipótesis o preguntas desde ángulos anteriormente ignorados. Lejos de cuestionar la validez u objetividad de la ciencia dominante, estas propuestas reconocen que los sistemas de conocimiento de las diferentes culturas «have different resources and limitations for producing 
knowledge; they are not all "equal," but there is no single possible perfect one, either» (Harding, 1998: 19). Muchos textos literarios se escriben y se estudian bajo este paraguas, lo cual nos permite detectar el modo en que los discursos patriarcales, clasistas, racistas o imperialistas se articulan y coconstruyen con la actividad científico-técnica. Veamos algunos ejemplos.

Los viajes científicos constituían «parte de la empresa colonial europea» (Asúa, 2004: 95). La catalogación, la sistematización, la nomenclatura de especies, la geografía y geología de otros espacios van conformando una percepción del Otro. Esta percepción configurará el mapa social, cultural y político europeo, y encontrará en la literatura un vehículo de expresión pero también de aplauso o de resistencia a sus significados. Resulta interesante comprobar cómo muchos textos literarios postcoloniales rescatan conceptos o prácticas que el discurso colonizador había asumido como irracionales, místicos, supersticiosos o mágicos, es decir, no científicos o pre-científicos, por ser diferentes al conocimiento compartido por la facción predominante. A modo de ejemplo, la caribeña Jean Rhys lo deja entrever en Ancho mar de Los Sargazos (1966), donde la mirada europea entiende el obeah como un sistema de creencias que atemoriza a los nativos, obviando el conocimiento de animales, plantas y minerales que implicaba y que también se empleaba con fines curativos, no sólo destructivos. De manera similar, el tiempo en la tradición mítica de algunas sociedades no se conceptualiza como lo hacemos en Occidente, donde la representación del tiempo tiene mucho que ver con el sistema capitalista (el tiempo es oro y tendemos a producir más en el menor tiempo posible), además de con las ideas de filósofos naturalistas de la antigüiedad y físicos de la actualidad. Por ejemplo, es más que frecuente encontrar un tiempo circular en las obras de los nativos norteamericanos, difícil de concebir si habitualmente imaginamos el tiempo como una línea que avanza hacia el futuro ${ }^{10}$. Parte de lo que denominamos realismo mágico en literaturas de países o sociedades anteriormente colonizados está relacionado con el alejamiento de parámetros cognitivos eurocéntricos que, a su vez, dieron lugar al género por excelencia de los imperios colonizadores, la novela realista. Colson Whitehead lo alegoriza en The Instuitionist (1999), una novela donde la protagonista, la primera mujer negra en convertirse en inspectora de ascensores en su ciudad (la lectura étnica sobre el ascenso

10 Martin Amis tuvo la bonita pero desafiante idea de escribir una novela donde los acontecimientos suceden hacia atrás en el tiempo. En La flecha del tiempo (1991), un experimento narrativo, pero también una exploración del pasado histórico, vemos cómo las uñas de los personajes crecen hacia adentro, las ciudades se demuelen progresivamente, llueve hacia arriba. 
social es evidente ${ }^{11}$ ), aplica un método poco convencional para la detección y resolución de problemas consistente en la comunicación no material con el ascensor, ciertamente alejado del método empirista empleado por la mayoría de inspectores de su entorno.

Entre las diversas causas que alejan a la mujer de la ciencia se encuentra la asociación entre literatura y feminidad a finales del siglo XVIII, cuando comenzó la brecha entre ambas prácticas: la ciencia, si quería ser ciencia por sí misma y consciente de sí misma, debía separarse de lo poético y metafísico, considerado «femenino» (Schiebinger, 1989: 159). Los movimientos feministas de los años 60 y 70 impulsaron la revisión de las relaciones entre ciencia y sociedad desde una perspectiva de género. Estas relaciones se establecieron a partir de una estructuración del mundo que incorporaba ideologías patriarcales que asociaban lo masculino a la razón, desplazando a las mujeres del logos. Francis Bacon, a quien se ha mencionado como uno de los iniciadores de la ciencia moderna, empleó metáforas sexuales para presentar a la naturaleza (el objeto de conocimiento) como femenina, y la actividad de conocer como masculina. Sin embargo, esta actividad no podía llevarse a cabo solo a través de la contemplación: la tarea exigía dominación (Lloyd, 1996; Merchant, 2001). Este vínculo entre ciencia y poder construye también una relación que refuerza el orden patriarcal de supremacía de lo masculino sobre lo femenino (la imagen de la joven «desentrañada» en la obra de Lautréamont analizada por Francisco González en este monográfico adquiere nuevos significados). Como con otras voces que tradicionalmente han sido apartadas de la creación de ciencia, resulta imposible detallar aquí los caminos que ha encontrado la literatura para exponer la desigualdad entre géneros inherente al discurso científico. Por mencionar solo algunos ejemplos, la anulación de la subjetividad femenina está ficcionalizada hasta el extremo en Las mujeres perfectas (1972), de Ira Levin, quien nos proporciona una fantasía patriarcal en la que los hombres de un pueblo asesinan a sus mujeres para reemplazarlas por androides sumisos que satisfacen todas sus necesidades. La ciencia médica también desempodera a la mujer en muchas narrativas decimonónicas, donde la figura del médico es, a menudo, tan importante como la del marido (Charles Bovary asume ambos roles en $\mathrm{Ma}$ dame Bovary, al igual que el marido de la narradora de «El empapelado

11 El novelista afro-norteamericano Walter Mosley publicó en The New York Times un artículo sobre la escasez de ciencia-ficción publicada por autores de etnias diferentes a la blanca, sugiriendo que estos autores han sido apartados de este espacio discursivo precisamente por la oportunidad de reescribir su historia y de imaginar un futuro alternativo: «For us science fiction offers an alternative where that which deviates from the norm is the norm» (2000: 405). 
amarillo», de Charlotte P. Gilman). La medicina, representada por el doctor, es un referente racional y autorizado cuando la mujer presenta comportamientos incompatibles con una feminidad adecuada. La ciencia ficción que especula con mundos lejanos en los que otro funcionamiento es posible ayuda a redefinir el concepto de género, por ejemplo en La mano izquierda de la oscuridad (1969), donde Ursula K. Le Guin imagina un planeta lejano cuyos habitantes no tienen un sexo fijo, pues se convierten en hombres o mujeres indistintamente durante el periodo de reproducción, y por ello no se relacionan en términos de diferencia ni jerarquía ni se interpretan en términos de género. El empoderamiento que supuso la aparición de la píldora anticonceptiva, donde la mujer había sido el objeto de la investigación química y médica, deriva en las series y novelas policiacas donde la científica se sitúa en el centro del discurso.

Muchas de las ansiedades generadas por la literatura fantástica, y también por la considerada más canónica, están relacionadas con la reproducción de la especie humana y, sobre todo, con la reproducción «antinatural» (es decir, por medio de maneras alternativas a la usual) que pueda amenazar órdenes patriarcales, o que pueda destruir el mismo orden científico-tecnológico que lo ha creado. La fantasía masculinista de apropiación del poder reproductor femenino se plasma en las historias de manipulación biológica (últimamente, genética). Ya Mary Shelley nos dio un toque de atención con el monstruo de Frankenstein, esa criatura viva y muerta, no nacida de mujer, cuyos fragmentos proceden de orígenes indeterminados y diversas clases sociales y que escapa, por tanto, a toda categorización. En el último tramo del siglo XX y ya en el XXI, tras el impacto cultural de las teorías de la relatividad y cuántica que redibujaron el mapa físico-cosmológico, estamos asistiendo al dibujo de un nuevo mapa interno, el biológico, que escudriña las estructuras celulares y moleculares. Cansados de preguntarnos si Dios existe, desplazamos la pregunta y cuestionamos si existimos nosotros en los términos en que habíamos pensado que existíamos, fijados en el siglo XVIII por las taxonomías de los naturalistas. Aunque el médico y botánico Linneo dejó muy claramente clasificado al homo sapiens en la décima edición de su Systema naturae de 1758, las revisiones sobre lo que define y cataloga al ser humano continúan hasta nuestros días, en especial con los estudios sobre biología molecular. En otras palabras, hasta qué punto somos humanos, y en qué punto dejamos de serlo, son inquietudes que se esconden detrás de la representación simbólica del miedo al Otro. Los planteamientos sobre lo prehumano, transhumano, posthumano y demás variantes enlazan con tecnologías e inquietudes investigadoras diversas: la robótica, la informática, la 
genética, las fuentes de energía alternativa, la química... La ciencia ficción y la literatura fantástica se alimentan con el desasosiego que nos produce perder nuestros límites humanos: convertirnos en zombis, alienígenas o vampiros (Drácula, de Bram Stoker, o Xenogenesis, de Olivia Butler); perder la corporeidad ante los demás (El hombre invisible, de H. G. Wells); compartir nuestro espacio corporal con otro (El extraño caso del Dr. Jeckyll y Mr. Hyde, de Robert L. Stevenson); involucionar hasta convertirnos en vegetales (La invasión de los ladrones de cuerpos, de Jack Finney); volver a la vida materializados en creaciones de un planeta pensante (Solaris, de Stanislaw Lem); evolucionar hasta convertirnos en máquinas («El hombre que se gastó», de Edgar A. Poe), etc.

Los textos literarios más especulativos desafían los supuestos de la Ilustración, que habían configurado la subjetividad humana como un yo orgánico, estable y unificado, y crean un sujeto «situated at an interface of communication, media and medical technology» (Goody, 2011: 161). Por ejemplo William Gibson, considerado el iniciador del cyberpunk, nos propone en «Johnny Mnemonic» (1981) o en Neuromancer (1984) la idea de mentes como circuitos computacionales que pueden ser alimentadas de información y recuerdos desde fuera de los términos corporales. El pensamiento se vuelve así tangible, material, público. Shelley Jackson, por su parte, lleva la fragmentación y recomposición identitaria aún más lejos en su hipertexto The Patchwork Girl (1995), donde la pantalla del ordenador se convierte en una mesa de disección como la que explora Francisco González en este mismo volumen, y donde la conectividad es multisemántica. La protagonista de Jackson es una criatura recompuesta, como el monstruo de Frankenstein, que (como un texto) es producto de una relación dinámica entre diferentes partes y discursos, incluidos los del lector/tecnousuario.

\section{CONTRIBUCIONES A ESTE MONOGRÁFICO}

Esta sección monográfica no trata de las distancias, sino de los encuentros. Este intento de «reunir lo que no está separado» (Sánchez Ron, 2011: 15) abarca diversas lenguas, diversos países, diversas maneras de explorar la literatura y de entenderla.

Jesús Pensado nos sitúa en una época donde la ciencia aún no existía como tal y la literatura como la entendemos ahora tampoco estaba definida, cuando el modo de conocer e interpretar el mundo se basaba en el humanismo y la religión. En su estudio contrastivo Pensado debate los tratados mé- 
dicos medievales como textos transnacionales y translingüísticos. Señala la importancia de estos textos en la creación del lenguaje científico en las lenguas peninsulares a través de la traducción y difusión de tratados y recetarios, que permitieron que la lengua vulgar encontrara expresión para términos que pertenecían en exclusiva a la lengua culta. Nos hace también reflexionar sobre los textos científicos de carácter divulgativo, un género que merece atención y reconocimiento por sí mismo y que cada vez encuentra más mercado en España ${ }^{12}$. No conviene olvidar que los tratados médicos posiblemente fueron fuente de inspiración para autores como Conan Doyle, autor analizado en el artículo de Manuel González de la Aleja. Pensado saca a la luz asimismo las habilidades retóricas de los creadores, practicantes o difusores de conocimiento. Este artículo, y el de Mariángel Soláns sobre el Alzheimer de Iris Murdoch, invitan a dos consideraciones importantes: la primera sobre la construcción cultural de la salud y la enfermedad ${ }^{13}$, y la segunda sobre la utilidad social de los textos ${ }^{14}$.

Carlos Miguel Pueyo nos traslada a la literatura decimonónica. Dos siglos antes de que cuestionáramos el concepto de «naturaleza» y lo resituáramos en el terreno de lo que los académicos hemos construido, deconstruido y reconstruido repetidamente, la comunidad poética del siglo XIX reclamaba su propia aportación al conocimiento de lo natural, pues vislumbró que la centralidad de la ciencia requería análisis y contestación. El artículo de Miguel Pueyo nos ofrece un ángulo de exploración que quiere alejarse de la tradicional mención de lo científico en literatura. Así, «[1]a ciencia en la literatura española decimonónica» propone acercarnos al modo en que los hallazgos de la ciencia (en este caso específico, el fenómeno de la luz) encuentran acomodo en el proceso creativo. Se ocupa este autor de «todo un repertorio de elementos atmosféricos que consideraban la luz como generadora de color y como elemento más destacado», una idea alimentada por la

12 En el mundo anglosajón algunos grandes nombres de la ciencia son también grandes divulgadores y encuentran sitio en las listas de bestsellers de no-ficción. Cosmos (1980) de Carl Sagan (en español en la editorial Planeta), A Brief History of Time (1988) de Stephen Hawkins (Historia del Tiempo: del Big Bang a los agujeros negros en Crítica) y Chaos: Making a New Science (1987) de James Gleick (Caos, también en Crítica) son ejemplos evidentes. El género no encuentra tanto acomodo en el mercado español, a pesar de contar con nombres destacados como Sánchez Ron.

13 «Podemos pasar por la vida sin saber que de una forma u otra nos hemos relacionado con los saberes físicos, químicos o matemáticos, pero nadie, ay, puede vivir en semejante inopia con respecto a la medicina, que nos acompaña de la cuna a la tumba» (Sánchez Ron: 2005: 8).

14 Resulta revelador el artículo de Montserrat Cabré, «Las prácticas de la salud en el ámbito doméstico: las recetas como textos de mujeres (s. XIV-XVII)», donde se trabaja con recetas medicinales escritas por mujeres en el ámbito doméstico que constituyen «una formulación simbólica ordenada a través del lenguaje [...] de un saber empírico» (2011: 27). 
obra Opticks, de Isaac Newton, y Zur Farbenlehre, del poeta-científico Goethe, además de otros tratados que estudiaban el interés de la luz y el espectro de colores a ella asociada. El artículo defiende que es la luz, entendida desde los estudios físicos del momento, el nexo de unión de ciencia y creatividad artística, un espacio de contacto por el que las «obras de arte total [...] se insertan en el camino de descubrimiento de la verdad de las cosas». La luz es, a fin de cuentas, el lenguaje del universo, un código de rayos y ondas mediante el cual nos hablan los elementos del cosmos y por el que podemos conocer su movimiento, propiedades, distancia o edad.

Margarita Rigal y Ricardo Marín nos introducen a la inquietud científica del siglo XIX, esos años en que la representación literaria de la tecno-ciencia nos mostró lo más iluminado y lo más oscuro del ser humano. Edgar Allan Poe, versado en matemáticas por formación y en otras disciplinas por devoción ${ }^{15}$, contribuyó enormemente a la difusión de la ciencia en Estados Unidos, no sólo por el contenido de sus relatos sino también por sus traducciones del francés. Es especialmente interesante que las técnicas inductivas de su detective, Dupin, se asemejen al método científico, testigo que recoge González de la Aleja en su propia contribución al volumen. Entre otros méritos, Poe dio forma al relato de anticipación científica, narraciones que muestran que «Poe era ducho en el arte de engañar al lector». Esta habilidad parte de sus estrategias narrativas, cómo no, pero también de la verosimilitud que aportaba a sus relatos, basada en sus lecturas sobre avances científico-tecnológicos y en lo que parecía el desarrollo esperable de la realidad norteamericana. El juego de verosimilitud en la narrativa de Poe nos plantea cuestiones como las de la representatividad literaria, o la responsabilidad ética del autor o autora en sus representaciones de la existencia. Este artículo rebate también aquellos comentarios sobre la obra de Poe que la tildan de escapista: Poe estaba muy en contacto con su mundo, y con cómo ese mundo variaba ante sus ojos.

Edgar Allan Poe y Jules Verne han quedado inscritos en la historia de la literatura como los escritores de la anticipación científica. Pilar Tresaco así nos lo confirma con su artículo, que nos pasea por la difusión de la obra de Verne en la prensa española decimonónica en conexión con el tema que aquí nos ocupa. Con notable frecuencia, Verne aparecía retratado en periódicos y revistas como un gran divulgador de la ciencia, recibiendo alabanzas por su vasto conocimiento de diversas materias científico-tecnológicas y por la

15 Ver García Lorenzo (2010). 
popularización de las mismas a través de sus novelas. En ocasiones, el encuentro con la ciencia en sus narraciones también despertaba disconformidad: estaban aquellos que criticaban su obra por poco literaria y también los que la encontraban demasiado fantasiosa. «La ciencia y Jules Verne en la prensa española del siglo XIX» nos aporta también la interesante idea de que las novelas de Verne constituían un referente «para todo aquello que implicara o supusiera una innovación, una osadía, un invento, una fantasía, un hecho científico y para todo aquello que la gente pudiera o quisiera idear o soñar». Si Verne ya lo había representado en sus novelas, la prensa las utilizaba para establecer comparaciones. Como creadora de iconos científicos la obra verniana es también impagable. Por ejemplo el Nautilus, a pesar de no ser el primer artefacto submarino en ser creado, se convirtió en referente para todos los que lo siguieron en la vida real.

Francisco González Fernández recurre a la anatomía y a las matemáticas en «Coser y cantar: la mesa de disección geométrica de Lautréamont», donde nos detalla la estética inherente a la disección anatómica, aunque a priori ambos conceptos nos puedan parecer antagónicos y de imposible reunión ${ }^{16}$. Este artículo nos lleva a considerar el escritorio como mesa de disección donde escritor y lector deben recomponer la experiencia humana, y donde el cuerpo es un texto que se puede leer, analizar, anotar, y corregir. En la obra Los Cantos de Maldoror (1869) Lautréamont reúne el lenguaje de la ciencia y el de la poesía «para injertar el primero en el segundo, buscando de este modo confeccionar una nueva poesía científica y matemática que estuviese en consonancia con los cambios epistemológicos que se habían producido en la era moderna». Como Pueyo en su artículo, no se trata de comprobar cómo se mencionan los adelantos científicos-tecnológicos de la época en unos textos determinados, sino que se destapa el cambio de modelo epistemológico del entorno, que empapa el proceso creativo literario. Al mismo tiempo, Francisco González lee el método matemático como «un auténtico escalpelo con el que vengarse del Creador desentrañando las verdades de la condición humana».

16 Podemos echar mano de la serie televisiva Da Vinci's Demons (Fox), donde un joven e impetuoso Leonardo da Vinci aparece sublimado por la belleza de la disección, no solo anatómica sino también mecánica: las partes y el todo forman un bello conjunto en cuanto son gráficamente expresados. También las series televisivas de las que nos habla González de la Aleja en su artículo, en especial CSI (CBS), nos remiten a la sublimación de la disección: la crudeza de las escenas de autopsia ha rebajado nuestro nivel de tolerancia, pero ha elevado nuestra fascinación por la significación de cada parte del cuerpo, tanto en su relación con el resto de partes como con la historia (qué ha sucedido) y el entorno (la escena del crimen) que los ha producido. 
Manuel González de la Aleja hace un repaso por el género detectivesco y la presencia de la ciencia en sus tramas desde sus comienzos, en forma de método analítico, pruebas policiales y exploraciones forenses. Su artículo «El caso del reactivo precipitado por la hemoglobina: la novela policíaca y sus (des)encuentros con la ciencia» abarca desde los primeros «detectives científicos» a los «científicos detectives» que hallamos en las más recientes novelas y series televisivas. Conviene no perder de vista que la aparición del género detectivesco en el siglo XIX está vinculada a la cultura científica del momento, la que apuesta por la exactitud metodológica y el uso de la razón (como vinculada está también con el nacimiento de la ciencia ficción, como apuntan Rigal y Marín en su artículo). Los propios relatos de Poe o Conan Doyle reflexionan, por boca de sus protagonistas, sobre cuestiones como el método analítico, el papel de la imaginación, las hipótesis de partida, o la validez de las deducciones. De Asúa nos recuerda que «[e]l prestigio de la ciencia como forma definitiva de conocimiento era muy alto en el siglo XIX y las constantes alusiones a los personajes, temas y métodos de la ciencia pudieron haber funcionado como un modo de legitimación del género para la audiencia contemporánea» (2004: 162). Puede que la autoridad del discurso científico contribuyera a la consolidación de este género popular, pero sin lugar a dudas el género contribuyó a su vez a la legitimación de la autoridad científica varias décadas más tarde. Esto es visible hoy en día en gran medida en las series televisivas con protagonistas pertenecientes a diversas disciplinas (medicina forense, paleontología, física teórica, bioquímica, etc.) cuyas rarezas personales en nada disminuyen su autoridad en la materia.

Por último, el artículo de Mariángel Soláns «"Navegando en la oscuridad": Iris Murdoch y la enfermedad de Alzheimer» explora la proyección social del Alzheimer, y los modos en que el discurso médico y el social hallan un punto de encuentro en la literatura. El diálogo que se establece entre ambos nos confirma que la literatura sí puede tener una utilidad, pues los investigadores «disponen de una extensa documentación que les permite avanzar en el conocimiento del desarrollo de esta enfermedad en su manifestación escrita». Este ensayo examina cómo los textos literarios se pueden convertir en fuente de datos para investigar problemas médicos, ya sea por la descripción de síntomas o procesos, o por las variaciones en el estilo del autor o autora. Además de constituirse en importante corpus para el estudio de los déficits lingüísticos durante la enfermedad de Alzheimer, el estudio de la obra de Murdoch desde este ángulo permite la reconstrucción del concepto de «enfermo/a de Alzheimer», despojando el término de parte del tabú con que se maneja hoy en día. Por último, el texto literario también puede 
convertirse en recurso educativo, como nos apunta Soláns en su trabajo, de forma que la literatura participe en la construcción de las ideas que tenemos sobre las cosas. «Literature is how we decide what matters to us» (Sleigh: 2011: 22), pues es a través de la literatura que podemos evaluar las implicaciones del discurso y la práctica científicos, negociar el poder de la certeza, valorar el mapa que nos presenta, aceptar sus limitaciones, temer sus posibilidades.

Mientras escribo estas líneas, en junio de 2013, se alinean dos acontecimientos: el «Manhattanhenge» ${ }^{17}$ y la muerte de Richard Matheson ${ }^{18}$. Ambos son vértices diferentes de la vastísima tierra liminal entre lo estético y lo científico-tecnológico, y prueban que, a medida que vamos desentrañando los secretos de lo visible e invisible, no podemos quedarnos sin nuestra ración de misterio e inconclusión:

Siempre había pensado en el mundo humano y en las dimensiones limitadas del hombre. Había sido presuntuoso con la naturaleza. Un centímetro era un concepto humano, no un concepto de la naturaleza. Para un hombre, cero centímetros significaba nada. Cero significaba nada.

Pero para la naturaleza, el cero no existía. La existencia continuaba en ciclos infinitos. ¡Ahora le parecía tan obvio! Jamás desaparecería, pues en el universo no había razones para no existir (Matheson, 2006: 248).

\section{REFERENCIAS BIBLIOGRÁFICAS}

ASÚA, Miguel de (2004). Ciencia y literatura: un relato histórico. Buenos Aires: EUDEBA.

BARTHES, R. (2009). «De la ciencia a la literatura». En El susurro del lenguaje: más allá de la palabra y la escritura. Trad. del francés por C. Fernández Medrano, 13-23. Barcelona: Paidós.

BEER, G. (1985) Darwin's Plots: Evolutionary Narrative in Darwin, George Eliot and Nineteenth Century Fiction. London: Ark.

17 Los estadounidenses han recogido y adaptado el fenómeno astronómico por el que el sol se alinea entre las piedras de Stonehenge en el solsticio de verano. En Manhattan, durante unos días antes del solsticio, el sol se alinea con las calles en cuadrícula y se producen auténticas concentraciones populares para contemplar el acontecimiento, convertido en conmovedor objeto estético.

18 Richard Matheson (1926-2013), escritor y guionista norteamericano, fue autor de novelas tan conocidas como Soy leyenda (I am Legend, 1954) y El increíble hombre menguante (The Shrinking Man, 1956). Los seguidores de series televisivas como The Twilight Zone o The Alfred Hitchcock Hour tal vez recuerden que también participó en la elaboración de guiones de algunos capítulos, basados en relatos suyos. 
BEER, G. (2010). «After Darwin's Plots.» Interdisciplinary Studies in the Long Nineteenth Century 11. http://www.19.bbk.ac.uk (último acceso: 1 agosto 2013).

BLANCO, R. (2001). «Guerras de la ciencia, imposturas intelectuales y estudios de la ciencia». REIS 94, 129-152. Versión digital: http://www. reis.cis.es/REIS/PDF/REIS_094_07.pdf (último acceso: 15 agosto 2013).

CABRÉ, M. (2011). «Las prácticas de la salud en el ámbito doméstico: las recetas como textos de mujeres (s. XIV-XVII)». En La mujer en la ciencia: historia de una desigualdad, Begoña Crespo et al., 25-41. Muenchen: LINCOM.

CALVINO, I. (1982). The Literature Machine: Essays. Trans. from the Italian by Patrick Creagh. London: Picador.

FRANKLIN, S. (1995). «Science as Culture, Cultures of Science». Annual Review of Anthropology 24, 163-184.

FOUCAULT, M. L. (1973). The Order of Things: An Archaeology of the Human Sciences. New York: Vintage Books.

FREESE, P. y HARRIS, C. (2004). The Holodeck in the Garden: Science and Technology in Contemporary American Literature. Illinois: Dalkey Archive.

GARCÍA ARANDA, José J. y GONZÁLEZ AGUDELO, Elvia M. (2007). «Entre la Literatura y las Ciencias Experimentales: hacia una mirada estética para el desarrollo didáctico de una cultura científica». Uni-pluri/ versidad 7, 1. Versión Digital. http://aprendeenlinea.udea.edu.co/revistas/index.php/unip/article/viewFile/11921/10803 (último acceso: 1 agosto 2013).

GARCÍA LANDA, J. A. (1991). «Science and Literature: Some Critical Parameters». En Science, Literature and Interpretations: Essays on Twentieth Century Literature and Critical Theory, Francisco Collado (ed.), 239-263. Zaragoza: Publicaciones de la Universidad.

GARCÍA LORENZO, M. M. (2010). «Edgar Allan Poe entre las artes y las ciencias». Revista de Creación Literaria Barcarola, 74/75, 251-261.

GOODY, A. (2011). Technology, Literature and Culture. Cambridge: Polity Press.

GROSS, P. y LEVITT, N. (1994). Higher Superstition: The Academic Left and its Quarrels with Science. Baltimore: Johns Hopkins University Press. 
HARDING, S. (1998). Is Science Multi-cultural?Postcolonialisms, Feminisms, and Epistemologies. Bloomington and Indianapolis: Indiana University Press.

HAYLES, K. N. (1989). «Chaos as Orderly Disorder: Shifting Ground in Contemporary Literature and Science». New Literary History 20. 2, 305322.

- (1990). Chaos Bound: Orderly Disorder in Contemporary Literature and Science. Ithaca: Cornell UP.

- (1994). «Deciphering the Rules of Unruly Disciplines: A Modest Proposal for Literature and Science». En Literature and Science, Donald Bruce and Anthony Purdy (eds.), 25-48. Amsterdam, Atlanta: Rodopi.

HEINLEIN, R. (1973). Time Enough for Love. New York: Penguin Putnam. KUNDERA, M. (1987). El arte de la novela. Barcelona: Tusquets.

LEAVIS, F. R. (1962). The Two Cultures? The Significance of C. P. Snow's Richmond Lecture. London: Chatto and Windus.

LLOYD, G. (1996). «Reason, Science and the Domination of Matter». En Feminism and Science, E. Fox Keller y H. Longino (eds.), 41-53. Oxford University Press.

MATHESON, Richard (2006). El increíble hombre menguante. Madrid: La Factoría de Ideas.

MERCHANT, C. (2001). «Dominion over Nature». En The Gender and Science Reader, M. Lederman y I. Bartsch (eds.), 68-81. London, New York: Routledge.

MERRISON, J. (1998). «The Death of the Poet: Coleridge and the Science of Logic». En The Third Culture: Literature and Science. European Cultures, vol. 9, Elinor S. Shaffer (ed.), 170-181. Berlin, New York: De Gruyter.

MOSLEY, W. (2000). «Black to the Future». En Dark Matter: A Century of Speculative Fiction From the African Diaspora, Sheree R. Thomas (ed.), 405-407. New York: Warner Books.

O'CONNOR, R. (2007). The Earth on Show. Chicago: University of Chicago Press.

PONTIN, J. (2007). «On Science Fiction: How it Influences the Imaginations of Technologists». MIT Technology Review. http://www.technologyreview.com/fromtheeditor/407393/on-science-fiction/ (último acceso: 21 agosto 2013). 
REEVES, C. (2005). The Language of Science. London, New York: Routledge.

RODRÍGUEZ MEDINA, M. (2013). «La ciencia de la literatura». La Red Información. http://laredinformacion.es/content/la-ciencia-de-la-literatura (último acceso: 21 agosto 2013).

SÁNCHEZ RON, J. M. (2000). «Literatura y ciencia». Letra Internacional 69, 2-4.

- (ed.) (2005). La ciencia y El Quijote. Barcelona: Crítica.

- (2011). La nueva Ilustración: Ciencia, Tecnología y Humanidades en un mundo interdisciplinar. Oviedo: Nobel.

— (2012). «La ciencia toma la ficción y... viceversa». El País. 2 marzo.

SHAFFER, Elinor S. (ed.) (1998). The Third Culture: Literature and Science. European Cultures, vol. 9. Berlin, New York: De Gruyter.

SCHIEBINGER, L. (1989). The Mind has no Sex? Women in the Origins of Modern Science. Cambridge, MA: Harvard University Press.

SLEIGH, C. (2011). Literature and Science. Basingstoke: Palgrave Macmillan.

SNOW, C. P. (1998). The Two Cultures. Cambridge: Cambridge University Press.

SOKAL, A. (1996a). «Transgressing the Boundaries: Towards a Transformative Hermeneutics of Quantum Gravity». Versión digital: http://www. physics.nyu.edu/faculty/sokal/transgress_v2/transgress_v2_singlefile. html (último acceso: 1 agosto 2013).

- (1996b). «A Physicist Experiments with Cultural Studies». Versión digital: http://www.physics.nyu.edu/faculty/sokal/lingua_franca_v4/lingua_ franca_v4.html (último acceso: 1 agosto 2013).

VALDÉS, M. J. y GUYON, E. (1998). «Serendipity in Poetry and Physics». En The Third Culture: Literature and Science. European Cultures, vol. 9, Elinor S. Shaffer (ed.), 28-39. Berlin, New York: De Gruyter.

VAN PEER, W. (1998). «Sense and Nonsense of Chaos Theory in Literary Studies». En The Third Culture: Literature and Science. European Cultures, vol. 9, Elinor S. Shaffer (ed.), 40-48. Berlin, New York: De Gruyter.

WOMACK, J. (2000). «Afterword». En Neuromancer, William Gibson, 265-276. New York: Ace. 
ZORPETTE, G. y ROSS, P. (2007). «The Books That Made A Difference». IEEE Spectrum. http://spectrum.ieee.org/geek-life/tools-toys/the-booksthat-made-a-difference (último acceso: 21 agosto 2013).

Recibido el 30 de junio de 2013.

Aceptado el 19 de septiembre de 2013. 